Originalien

Psychotherapeut 2020 $65: 398-404$ https://doi.org/10.1007/s00278-020-00442-w Online publiziert: 19. August 2020

๑) Der/die Autor(en) 2020

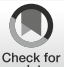

\author{
Alexander Obbarius ${ }^{1} \cdot$ Lea Sarrar ${ }^{2,3}$ \\ 'Centrum für Innere Medizin und Dermatologie, Medizinische Klinik mit Schwerpunkt Psychosomatik, \\ Charité - Universitätsmedizin Berlin, Berlin, Deutschland \\ ${ }^{2}$ Fakultät Naturwissenschaften, Department Psychologie, MSB Medical School Berlin, Berlin, Deutschland \\ ${ }^{3}$ Klinik für Audiologie und Phoniatrie, Charité - Universitätsmedizin Berlin, Berlin, Deutschland
}

\title{
Selbstberichtete anorektische Essstörungssymptomatik und psychische Struktur
}

\section{Zusammenhang bei weiblichen Adoleszenten und jungen Erwachsenen in einer Onlinestichprobe}

\section{Zusatzmaterial online \\ Zusätzliche Informationen sind in der Onlineversion dieses Artikels (https://doi.org/ 10.1007/s00278-020-00442-w) enthalten.}

\section{Essstörungen gelten als schwer- wiegende Erkrankungen mit hohen Chronifizierungsraten und langen Behandlungszeiten (Briegel et al. 2018). Auch subklinische Formen von Essstörungen bergen ein ho- hes Risiko für die Entwicklung einer manifesten Erkrankung, weshalb eine frühzeitige Diagnostik und Untersuchung von auffälligem Ess- verhalten und zugrunde liegenden Faktoren sinnvoll sind (Fichter 2015). Ein bedeutsamer Aspekt während der psychodynamischen Diagnostik und Behandlungsplanung stellt bei Patienten' ${ }^{1}$ mit Essstörungen, eben- so wie bei Patienten mit anderen psychischen Erkrankungen, die Er- fassung der psychischen Struktur dar.}

1 Sofern nicht anders gekennzeichnet, bezieht sich die Nennung des männlichen Geschlechts auf alle Geschlechter.

\section{Theoretischer Hintergrund}

Die aktuelle Operationalisierte Psychodynamische Diagnostik (OPD) definiert Struktur als die „Verfügbarkeit über psychische Funktionen in der Regulierung des Selbst und seiner Beziehung zu den inneren und äußeren Objekten" (Arbeitskreis OPD 2014, S. 225). Zwischen dem psychodynamischen Strukturbegriff und Persönlichkeitsaspekten sowie Persönlichkeitsstörungen bestehen große Überschneidungen (Spitzer 2004).

Bei Patienten mit Essstörungen wurden bereits bestimmte akzentuierte Persönlichkeitseigenschaften beschrieben. So zeigen sich Patienten mit Anorexia nervosa häufig introvertiert, konfliktvermeidend und perfektionistisch (Dahlenburg et al. 2019). Zudem offenbaren sich bei ihnen ein geringer Selbstwert und eine eingeschränkte Emotionsregulationsfähigkeit (Hartmann et al. 2014). Ferner berichten Studien über ein unsicheres Bindungsverhalten (Pace et al. 2016). Zu dem Zusammenhang zwischen Essstörungen, deren Ausprägung und OPDStruktur liegen bisher allerdings sehr wenige empirische Befunde vor. In einer Studie an 60 erwachsenen Patienten mit Essstörungen wurden die Werte einer Kontrollgruppe deutlich übertroffen (Rohde et al. 2019; Ehrenthal et al. 2012). Einige Studien belegen den Zusammen- hang zwischen psychischen Störungen und strukturellen Funktionen - auch bei Adoleszenten, wobei davon ausgegangen wird, dass Einschränkungen der strukturellen Funktionen das Risiko für die Entwicklung von psychischen Störungen erhöht (Huber et al. 2017). Bisher liegen jedoch keine spezifischen Untersuchungen zur psychischen Struktur bei Adoleszenten und jungen Erwachsenen mit Essstörungen vor, obwohl dies für die Einordnung klinischer Befunde bei diesen Patienten wünschenswert wäre.

Das Ziel dieser Studie besteht daher in der Beschreibung struktureller Funktionen bei Adoleszenten und jungen Erwachsenen mit unterschiedlicher Ausprägung von Essstörungssymptomatik. Es wird erwartet, dass sich die selbstberichtete OPD-Struktur zwischen 3 Gruppen mit keiner, geringer und ausgeprägter selbstberichteter Essstörungssymptomatik unterscheidet.

\section{Material und Methoden}

\section{Stichprobe}

Die Probandinnen wurden 2018 online auf sozialen Plattformen (Facebook und Instagram) rekrutiert. Darunter befanden sich auch Foren oder Profile, die sich mit den Themen Körperideale, Essstörungen oder Abnehmen beschäftigten. 
Tab. 1 Kerndiagnosekriterien der Anorexia nervosa (ICD-10) und zugehörige adaptierte Fragen bzw. Items in Anlehnung an das SIAB

\begin{tabular}{|c|c|c|}
\hline $\begin{array}{l}\text { Kerndiagnosekriterien einer Anorexia nervosa } \\
\text { gemäß ICD-10 }\end{array}$ & Verwendete Fragen/Items in Anlehnung an das SIAB & $\begin{array}{l}\text { Kerndiagnosekriterium } \\
\text { erfüllt, wenn ... }\end{array}$ \\
\hline $\begin{array}{l}\text { Kriterium A } \\
\text { Gewichtsverlust oder fehlende Gewichtszunahme }\end{array}$ & $\begin{array}{l}\text { 1. Was ist Deine/lhre derzeitige Körpergröße (in Metern)? } \\
\text { 2. Was ist Dein/lhr derzeitiges Gewicht (in Kilogramm)? } \\
\text { 3. Ich habe in letzter Zeit viel Gewicht verloren (ja/nein) }\end{array}$ & $\begin{array}{l}\text { 1. und 2.: } \mathrm{BMI}<17,5 \mathrm{~kg} / \mathrm{m}^{2} \\
\text { und } 3 .: \mathrm{Ja}\end{array}$ \\
\hline $\begin{array}{l}\text { Kriterium B } \\
\text { Selbst herbeigeführter Gewichtsverlust durch die } \\
\text { Vermeidung „fettmachender" Speisen }\end{array}$ & $\begin{array}{l}\text { 1. Der Gewichtsverlust war selbst herbeigeführt, beispielswei- } \\
\text { se durch das Vermeiden von „fettmachenden“ Speisen (ja/ } \\
\text { nein) }\end{array}$ & 1.: Ja \\
\hline $\begin{array}{l}\text { Kriterium C } \\
\text { Selbstwahrnehmung als „zu fett”, verbunden mit } \\
\text { einer sich aufdrängenden Furcht, zu dick zu werden, } \\
\text { niedrige Gewichtsschwelle }\end{array}$ & $\begin{array}{l}\text { 1. Ich finde mich selbst zu fett und habe Angst, zu dick zu } \\
\text { werden (ja/nein) } \\
\text { 2. Ich habe ein Wunschgewicht (ja/nein) } \\
\text { 3. Welches (in Kilogramm)? }\end{array}$ & $\begin{array}{l}\text { 1. oder 2.: Ja oder } 3 \text {. (unter } \\
\text { Berücksichtigug von Kriteri- } \\
\text { um A 1.): BMI }<17,5 \mathrm{~kg} / \mathrm{m}^{2}\end{array}$ \\
\hline $\begin{array}{l}\text { Kriterium D } \\
\text { Umfassende endokrine Störung der Achse Hypothala- } \\
\text { mus-Hypophyse-Gonaden (Amenorrhö) }\end{array}$ & $\begin{array}{l}\text { 1. Ich habe meine Regelblutung (ja/nein, hatte ich noch nie/ } \\
\text { nein, nicht mehr) }\end{array}$ & $\begin{array}{l}\text { 1.: Nein, hatte ich noch nie } \\
\text { oder } \\
\text { Nein, nicht mehr }\end{array}$ \\
\hline
\end{tabular}

Die Daten wurden elektronisch mithilfe der Analyseplattform QuestionPro (www.questionpro.com/de) erhoben. Vor der Befragung wurden die Probandinnen über die Studie, die Freiwilligkeit der Teilnahme sowie den Datenschutz informiert. Per Button musste bestätigt werden, dass die Aufklärung gelesen und verstanden wurde sowie das Einverständnis zur Studienteilnahme erfolgt. Bei Minderjährigen wurde zusätzlich das Einverständnis der Eltern erfragt, indem per Klick bestätigt wurde, dass die Eltern die Studienaufklärung gelesen und einer Teilnahme zugestimmt haben. Da das Ziel der vorliegenden Studie in der Untersuchung von anorektischer Essstörungssymptomatik und psychischer Struktur bei Adoleszenten bzw. jungen Erwachsenen lag, wurden als Einschlusskriterien ein Alter zwischen 14 und 25 Jahren sowie ein BodyMass-Index (BMI) im unter- oder im normalgewichtigen Bereich festgelegt. Insgesamt beantworteten 167 Probandinnen den kompletten Fragebogen, wobei 24 aufgrund eines BMI $\geq 25 \mathrm{~kg} / \mathrm{m}^{2}$ aus den Analysen ausgeschlossen wurden.

\section{Diagnostische Verfahren}

\section{Strukturiertes Inventar für Anorektische und Bulimische \\ Essstörungen}

Das Strukturierte Inventar für Anorektische und Bulimische Essstörungen (SIAB; Fichter und Quadflieg 1999) dient der Diagnostik klinischer Essstörungen. In der vorliegenden Studie wurden Fragen bzw. Items zu den Kerndiagnosekriterien einer Anorexia nervosa gemäß Internationaler Klassifikation psychischer Störungen (ICD-10; Dilling et al. 2011) sowie in Anlehnung an das SIAB verwendet und per Selbstbericht von den Probanden beantwortet (• Tab. 1). Für den Zweck der vorliegenden Studie wurden die Probandinnen im Anschluss in die 3 Gruppen „keine anorektische Essstörungssymptomatik“" (0 von 4 Kriterien; „K-AN“), ,geringe anorektische Essstörungssymptomatik" (1-2 von 4 Kriterien; „G-AN“) und „ausgeprägte anorektische Essstörungssymptomatik“ (3-4 von 4 Kriterien; „A$\mathrm{AN}^{\text {“) }}$ unterteilt. Probandinnen mit einer Vordiagnose einer Essstörung wurden von der ersten Gruppe ausgeschlossen $(n=2)$. Die beiden letzten Gruppen wurden im Kontext dieser Studie als subklinische Formen von Essstörungen aufgefasst, da mindestens ein Symptom der Kerndiagnosekriterien einer Anorexia nervosa gemäß ICD-10 (Dilling et al. 2011) erfüllt war. Psychometrische Daten des SIAB hinsichtlich der Reliabilität, der internen Konsistenz der Subskalen und der konvergenten Validität können als gut betrachtet werden (Fichter und Quadflieg 1999).

\section{Eating Disorder Inventory}

Zur Erhebung essstörungsspezifischer und allgemeiner Psychopathologie wurde die gekürzte Version des Eating Dis- order Inventory (EDI-2; Paul und Thiel 2005) verwendet. Es erfasst 8 Skalen („Schlankheitsstreben“, „Bulimie“, „Unzufriedenheit mit dem Körper“, „Ineffektivität“, „Perfektionismus", „Misstrauen“, „Interozeptive Wahrnehmung“, „Angst vor dem Erwachsenwerden"). Psychometrische Daten hinsichtlich Reliabilität und Validität des EDI-2 können als befriedigend bis gut betrachtet werden (Kappel et al. 2012).

\section{Fragebogen zur Strukturachse der Operationalisierten Psychodynamischen Diagnostik}

Zur Erhebung der psychischen Struktur wurde der Fragebogen zur Strukturachse der Operationalisierten Psychodynamischen Diagnostik verwendet (OPDSF; Ehrenthal et al. 2012). Der Fragebogen umfasst 8 Hauptskalen, mit denen 21 der 24 Subskalen der OPD-Strukturachse abgedeckt werden können (siehe Zusatzmaterial Tab. 1 in Ehrenthal et al. 2012). Zusätzlich wird ein Gesamtwert der Struktur über alle Skalen hinweg gebildet. Hohe Werte sprechen für eine hohe Beeinträchtigung der psychischen Struktur. Die interne Konsistenz der Skalen beträgt zwischen $\alpha=0,72$ und $\alpha=0,91$ (Cronbachs $\alpha$ ) und kann demnach als befriedigend bis sehr gut eingeschätzt werden. Der OPD-SF ist zur Anwendung ab einem Alter von 13 Jahren geeignet (Bock et al. 2018). 
Psychotherapeut 2020 - 65:398-404 https://doi.org/10.1007/s00278-020-00442-w

(c) Der/die Autor(en) 2020

\section{A. Obbarius · L. Sarrar}

\section{Selbstberichtete anorektische Essstörungssymptomatik und psychische Struktur. Zusammenhang bei weiblichen Adoleszenten und jungen Erwachsenen in einer Onlinestichprobe}

\section{Zusammenfassung}

Hintergrund. Bei verschiedenen psychischen Störungsbildern, wie beispielsweise Essstörungen, zeigen sich Einschränkungen in der psychischen Struktur, die sowohl bei Erwachsenen als auch Adoleszenten beschrieben wurden. Bisher liegen jedoch keine empirischen Untersuchungen zur psychischen Struktur bei Adoleszenten bzw. jungen Erwachsenen mit typischen oder subklinischen Formen von Essstörungen vor. Ziele der Arbeit. Die vorliegende Studie zielt auf die Untersuchung und den Vergleich verschiedener Strukturdimensionen gemäß Operationalisierter Psychodynamischer Diagnostik bei weiblichen Adoleszenten und jungen Erwachsenen mit unterschiedlicher Ausprägung selbstberichteter anorektischer Essstörungssymptomatikab.

Material und Methoden. Es gingen Daten von 141 weiblichen Adoleszenten und jungen Erwachsenen mit keiner, gering oder ausgeprägter selbstberichteter anorektischer Essstörungssymptomatik ein. Die Probandinnen wurden über die sozialen Netzwerke Facebook und Instagram rekrutiert und mithilfe einer Onlineerhebung befragt. Zum Einsatz kamen der Fragebogen zur Strukturachse der Operationalisierten Psychodynamischen Diagnostik (OPD-SF) sowie das Eating Disorder Inventory-2 (EDI-2). Ergebnisse. Adoleszente und junge Erwachsene mit ausgeprägter selbstberichteter anorektischer Essstörungssymptomatik zeigen auf fast allen Skalen des OPD-SF sowie auf sämtlichen Skalen des EDI-2 signifikant höhere Werte als Adoleszente und junge Erwachsene ohne bzw. mit geringer Symptomatik. Diese Unterschiede bleiben auch nach Kontrolle für das Alter sowie Vorliegen von Vordiagnosen und psychotherapeutischen
(Vor)Behandlungen bestehen. Es zeigen sich deutliche Zusammenhänge zwischen den Skalen des OPD-SF und denen des EDI-2. Schlussfolgerung. Einschränkungen in der psychischen Struktur scheinen sich bereits bei subklinischen Formen gestörten Essverhaltens zu zeigen, zumindest, wenn diese selbstberichtet ist. Die Ergebnisse unterstützen die Notwendigkeit der frühzeitigen Erkennung von Problemen mit dem Essverhalten und des Einbezugs struktureller Aspekte in die psychotherapeutische Behandlung.

Schlüsselwörter

Anorexia nervosa · Persönlichkeitsstruktur . Selbstwahrnehmung · Operationalisierte Psychodynamische Diagnostik · Soziale Netzwerke

\section{Self-reported symptoms of anorectic eating disorders and personality structure. Association in female adolescents and young adults in an online sample}

\section{Abstract}

Background. Impairments in the personality structure are present in various mental disorders, such as eating disorders and these impairments have been described in both adults and adolescents; however, so far there have been no empirical investigations on personality structure in adolescents or young adults with typical or subclinical forms of eating disorders.

Objective. The aim of this study was to investigate and compare various structural dimensions among female adolescents and young adults with different severities of self-reported symptoms of anorectic eating disorders.

Material and methods. Data from 141 female adolescents and young adults without, with minor, and with major self-reported symptoms of anorectic eating disorders were included in this study. The participants were recruited on the social network platforms Facebook and Instagram and asked to complete an online survey. The operationalized psychodynamic diagnosis structural questionnaire (OPD-SQ) system and the eating disorder inventory-2 (EDI-2) were administered.

Results. Adolescents and young adults with self-reported symptoms of major anorectic eating disorders demonstrated higher scores on almost all scales of the OPD-SQ and all scales of the EDI-2 than adolescents and young adults with minor or no symptoms of eating disorders. These differences persisted even after controlling for age, previous diagnoses and (prior) psychotherapeutic treatment. In addition, a strong correlation was found between the OPD-SQ and the EDI-2 scales in the sample.

Conclusion. Impairment in personality structure seems to be present even in subclinical conditions of eating disorders, at least when they are self-reported. These results support the need for early detection of problematic eating behavior and the inclusion of structural aspects in psychotherapeutic interventions.

\section{Keywords}

Anorexia nervosa - Personality structure - Selfperception - Operationalized psychodynamic diagnosis · Social media

\section{Statistische Datenanalyse}

Die Stichprobencharakteristika (Alter, BMI) der 3 Gruppen (K-AN, G-AN, A-AN) wurden mithilfe einfaktorieller univariater Varianzanalysen (ANOVAs) verglichen. Häufigkeitsanalysen bezüglich möglicher Vordiagnosen und (aktueller und/oder vergangener) psycho- therapeutischer Behandlungen erfolgten mithilfe des $\chi^{2}$-Tests sowie paarweisen Post-hoc-Tests. Gruppenunterschiede hinsichtlich der allgemeinen und der essstörungsspezifischen Psychopathologie wurden mithilfe von ANOVAs (Skalen des EDI-2 als abhängige Variablen) berechnet, „da die einzelnen Subskalen relativ unabhängige Merkma- le widerspiegeln“ (Paul und Thiel 2005 S. 16). Für den OPD-SF kam/en eine einfaktorielle univariate Kovarianzanalyse (ANCOVA, Gesamtwert des OPD-SF als abhängige Variable) bzw. multivariate Kovarianzanalyse für die Strukturdimensionen des OPD-SF (MANCOVA, Skalen des OPD-SF als abhängige Variablen) zum Einsatz, wobei das Alter sowie 


\begin{tabular}{|c|c|c|c|c|c|c|c|c|}
\hline & & \multirow[t]{2}{*}{ K-AN $(n=38)$} & \multirow[t]{2}{*}{ G-AN $(n=68)$} & \multirow[t]{2}{*}{ A-AN $(n=35)$} & \multicolumn{3}{|c|}{ ANOVA $(d f=2)$} & \multirow[t]{2}{*}{ Post-hoc } \\
\hline & & & & & $\mathbf{F}$ & $p$ & $\eta 2_{\text {part }}$ & \\
\hline \multirow{3}{*}{$\begin{array}{l}\text { Alter } \\
\text { (Jahre) }\end{array}$} & M & 21,27 & 20,31 & 18,29 & \multirow[t]{3}{*}{9,96} & \multirow[t]{3}{*}{$<0,001^{* * *}$} & \multirow[t]{3}{*}{0,127} & \multirow[t]{3}{*}{$\mathrm{K}-\mathrm{AN}=\mathrm{G}-\mathrm{AN}<\mathrm{A}-\mathrm{AN}$} \\
\hline & SD & $\pm 3,05$ & $\pm 2,91$ & $\pm 2,74$ & & & & \\
\hline & $95 \%-K I$ & $20,25-22,29$ & $19,60-21,01$ & $17,34-19,23$ & & & & \\
\hline \multirow[t]{3}{*}{ BMI } & M & 21,36 & 20,64 & 16,70 & \multirow[t]{3}{*}{55,15} & \multirow[t]{3}{*}{$<0,001^{* * *}$} & \multirow[t]{3}{*}{0,444} & \multirow[t]{3}{*}{$\mathrm{K}-\mathrm{AN}=\mathrm{G}-\mathrm{AN}<\mathrm{A}-\mathrm{AN}$} \\
\hline & SD & $\pm 1,72$ & $\pm 2,40$ & $\pm 1,72$ & & & & \\
\hline & $95 \%-K I$ & $20,77-21,93$ & $20,06-21,22$ & $16,11-17,29$ & & & & \\
\hline \multirow[t]{2}{*}{-} & & \multirow[t]{2}{*}{ K-AN $(n=38)$} & \multirow[t]{2}{*}{ G-AN $(n=67)$} & \multirow[t]{2}{*}{ A-AN $(n=35)$} & \multicolumn{3}{|c|}{$X^{2}(d f=2)$} & \multirow[t]{2}{*}{ Post-hoc } \\
\hline & & & & & $x^{2}$ & \multicolumn{2}{|l|}{$p$} & \\
\hline \multicolumn{2}{|c|}{ Vordiagnosen $^{\mathrm{a}}$} & $\begin{array}{l}15,79 \% \\
(n=6)\end{array}$ & $\begin{array}{l}37,35 \% \\
(n=39)\end{array}$ & $\begin{array}{l}85,71 \% \\
(n=30)\end{array}$ & 36,69 & \multicolumn{2}{|l|}{$<0,001^{* * *}$} & $\mathrm{~K}-\mathrm{AN}<\mathrm{G}-\mathrm{AN}<\mathrm{A}-\mathrm{AN}$ \\
\hline \multicolumn{2}{|c|}{$\begin{array}{l}\text { Psychotherapeutische } \\
\text { (Vor)Behandlungen }\end{array}$} & $\begin{array}{l}36,32 \% \\
(n=10)\end{array}$ & $\begin{array}{l}63,24 \% \\
(n=43)\end{array}$ & $\begin{array}{l}85,71 \% \\
(n=30)\end{array}$ & 27,58 & \multicolumn{2}{|l|}{$<0,001^{* * *}$} & $\mathrm{~K}-\mathrm{AN}<\mathrm{G}-\mathrm{AN}<\mathrm{A}-\mathrm{AN}$ \\
\hline \multicolumn{9}{|c|}{$\begin{array}{l}\text { K-AN keine selbstberichtete (anorektische) Essstörungssymptomatik, G-AN geringe selbstberichtete (anorektische) Essstörungssymptomatik, } A \text {-AN aus- } \\
\text { geprägte selbstberichtete (anorektische) Essstörungssymptomatik, ANOVA „analysis of variance“, } M \text { Mittelwert, SD Standardabweichung, } 95 \% \text {-KI } 95 \% \text { - } \\
\text { Konfidenzintervall, } d f \text { Freiheitsgrade, } F \text { bzw. X Prüfgröße, } \eta 2 \text { part Effektstärke, partielles Eta-Quadrat, } p=\text { Signifikanz } \\
{ }^{2} \text { Das Vorliegen einer Vordiagnose einer Essstörung wurde in der Gruppe K-AN ausgeschlossen } \\
* * * * 0<0,001\end{array}$} \\
\hline
\end{tabular}

Vorliegen von Vordiagnosen und psychotherapeutischen (Vor)Behandlungen als Kovariaten berücksichtigt wurden. Diese Aspekte wurden auf der Grundlage vorhandener Literatur als mögliche Einflussvariablen auf die OPD-Struktur identifiziert (Arbeitskreis OPD-KJ-2 2014; Spitzer 2004). Sämtliche varianzanalytischen Berechnungen wurden einer Post-hoc-Analyse unter Zuhilfenahme des Tukey-B-Verfahrens unterzogen, sodass einer - im Rahmen vom multiplen Testen zu erwartenden - $a$-FehlerInflation begegnet wurde (Field 2013). Die Voraussetzungen für die Durchführung varianzanalytischer Verfahren waren erfüllt (insbesondere hinreichende Normalverteilung, Homoskedastizität, Linearität, keine Ausreißer; Field 2013). Das Effektstärkemaß (ES) wurde mithilfe des partielles Eta-Quadrats ( $\eta 2_{\text {part }}$ ) angegeben; zudem wurden die jeweiligen Konfidenzintervalle berechnet. Die Klassifikation der ES erfolgte für $\eta 2_{\text {part }}$ mit $\eta 2_{\text {part }}=0,01$ klein, $\eta 2_{\text {part }}=0,06$ mittel und $\eta 2_{\text {part }}=0,14$ groß. In einem weiteren Schritt wurden korrelative Zusammenhänge zwischen dem EDI-2 und OPDSF untersucht. Die genannten Kovariaten wurden abermals im Rahmen einer Partialkorrelation berücksichtigt. Die Korrelationshöhen wurden wie folgt eingeordnet: $r=0,1$ gering, $r=0,3$ mittel und $r=0,5$ hoch. Die Interpretation sämt- licher ES erfolgte nach Cohen (1988). Das Signifikanzniveau wurde a priori auf $\alpha=0,05$ festgelegt. Die statistischen Berechnungen erfolgten mithilfe von IBM SPSS (Version 25.0).

\section{Ergebnisse}

\section{Deskriptive Ergebnisse}

38 Probandinnen wiesen keines der Kerndiagnosekriterien einer Anorexia nervosa auf und fielen damit in die Gruppe „K-AN“, 68 Probandinnen erfüllten eines bis zwei der Kerndiagnosekriterien und wurden damit der Gruppe „GAN" zugeordnet und 35 Probandinnen erfüllten drei bis vier der Kerndiagnosekriterien und zählten damit zur Gruppe „A-AN“. Die drei Gruppen unterschieden sich signifikant hinsichtlich Alter und BMI sowie dem Vorliegen von Vordiagnosen und psychotherapeutischen (Vor)Behandlungen bei großen Effektstärken für Alter und BMI (• Tab. 2). Erwartungsgemäß ergaben sich darüber hinaus auf sämtlichen Skalen des EDI-2 signifikante Gruppenunterschiede mit durchweg großen Effekten bei einer Spannweite von $\eta 2_{\text {part }}=, 151$ bis ,614 (Zusatzmaterial online: Tab. 4).

\section{Psychische Struktur}

Das MANCOVA-Modell der OPD-SFSkalen offenbarte signifikante Unterschiede zwischen den 3 Gruppen (F (16, $254)=2,557 ; \quad p=0,001 ; \quad \eta 2_{\text {part }}=0,139$; Wilk's $\lambda=0,742)$. Auch nach Kontrolle der Kovariaten ergaben sich auf den einzelnen Skalen der Strukturdimensionen des OPD-SF sowie bezüglich des Gesamtwerts deutliche Unterschiede zwischen den 3 Gruppen mit durchweg signifikanten Befunden. Eine Ausnahme bildete die Strukturdimension Regulierung des Objektbezugs. Hierbei ergaben sich nach Kontrolle der Kovariaten keine signifikanten Unterschiede zwischen den Gruppen. Die Gruppe A-AN zeigte auf sämtlichen berichteten Skalen und dem Gesamtwert des OPD-SF höhere Werte. Es wurden kleine bis große Effektstärken deutlich, die sich zwischen $\eta 2_{\text {part }}=0,044$ (Objektwahrnehmung) und 0,139 (Bindungsfähigkeit an innere Objekte) bewegten (• Tab. 3; Zusatzmaterial online: Abb. 1).

\section{Zusammenhang zwischen essstörungsspezifischer sowie allgemeiner Psychopathologie und psychischer Struktur}

Sämtliche Skalen des OPD-SF standen auch nach Kontrolle der Kovariaten - in 
Tab. 3 Psychische Struktur (OPD-SF; MANCOVA [Skalen des OPD-SF] bzW. ANCOVA [Gesamtwert des OPD-SF]); Kovariaten: Alter, Vordiagnose, psychotherapeutische [Vor]Behandlungen)

\begin{tabular}{|c|c|c|c|c|c|c|c|c|}
\hline & & \multirow{2}{*}{$\begin{array}{l}\text { K-AN } \\
(n=38)\end{array}$} & \multirow{2}{*}{$\begin{array}{l}\text { G-AN } \\
(n=68)\end{array}$} & \multirow{2}{*}{$\begin{array}{l}\text { A-AN } \\
(n=35)\end{array}$} & \multicolumn{3}{|c|}{ MANCOVA bzw. ANCOVA (OPD-Gesamtwert) $(d f=2)$} & \multirow[t]{2}{*}{ Post-hoc } \\
\hline & & & & & $\mathbf{F}$ & $p$ & $\eta 2_{\text {part }}$ & \\
\hline \multirow[t]{3}{*}{ Selbstwahrnehmung ${ }^{\mathrm{b}}$} & M & 1,63 & 2,35 & 2,92 & \multirow[t]{3}{*}{6,31} & \multirow[t]{3}{*}{$0,002^{* *}$} & \multirow[t]{3}{*}{0,086} & \multirow{3}{*}{$\begin{array}{l}\mathrm{K}-\mathrm{AN}<\mathrm{G}- \\
\mathrm{AN}<\mathrm{A}-\mathrm{AN}\end{array}$} \\
\hline & SD & $\pm 0,61$ & $\pm 0,83$ & $\pm 0,60$ & & & & \\
\hline & $95 \%-K I$ & $1,42-1,83$ & $2,15-2,55$ & $2,72-3,13$ & & & & \\
\hline \multirow[t]{3}{*}{ Objektwahrnehmung ${ }^{\mathrm{b}}$} & M & 2,01 & 2,38 & 2,75 & \multirow[t]{3}{*}{3,12} & \multirow[t]{3}{*}{$0,048^{*}$} & \multirow[t]{3}{*}{0,044} & \multirow{3}{*}{$\begin{array}{l}\mathrm{K}-\mathrm{AN}<\mathrm{G}- \\
\mathrm{AN}<\mathrm{A}-\mathrm{AN}\end{array}$} \\
\hline & SD & $\pm 0,45$ & $\pm 0,59$ & $\pm 0,60$ & & & & \\
\hline & $95 \%-K I$ & $1,86-2,16$ & $2,24-2,53$ & $2,54-2,95$ & & & & \\
\hline \multirow[t]{3}{*}{ Selbstregulierung $\mathrm{a}, \mathrm{c}$} & M & 1,76 & 2,50 & 2,89 & \multirow[t]{3}{*}{6,31} & \multirow[t]{3}{*}{$0,002^{* *}$} & \multirow[t]{3}{*}{0,086} & \multirow{3}{*}{$\begin{array}{l}\mathrm{K}-\mathrm{AN}<\mathrm{G}- \\
\mathrm{AN}<\mathrm{A}-\mathrm{AN}\end{array}$} \\
\hline & SD & $\pm 0,61$ & $\pm 0,74$ & $\pm 0,57$ & & & & \\
\hline & $95 \%-K I$ & $1,57-1,96$ & $2,32-2,68$ & $2,69-3,08$ & & & & \\
\hline \multirow{3}{*}{$\begin{array}{l}\text { Regulierung } \\
\text { d. Objektbezugs }\end{array}$} & M & 1,93 & 2,26 & 2,50 & \multirow[t]{3}{*}{0,51} & \multirow[t]{3}{*}{0,604} & \multirow[t]{3}{*}{0,007} & \multirow[t]{3}{*}{ / } \\
\hline & SD & $\pm 0,60$ & $\pm 0,66$ & $\pm 0,64$ & & & & \\
\hline & $95 \%-K I$ & $1,73-2,13$ & $2,10-2,42$ & $2,27-2,71$ & & & & \\
\hline \multirow{3}{*}{$\begin{array}{l}\text { Emotionale } \\
\text { Kommunikation nach }^{\text {innen }}{ }^{b}\end{array}$} & M & 1,86 & 2,21 & 2,87 & \multirow[t]{3}{*}{8,89} & \multirow[t]{3}{*}{$<0,001^{* * * *}$} & \multirow[t]{3}{*}{0,117} & \multirow{3}{*}{$\begin{array}{l}\mathrm{K}-\mathrm{AN}<\mathrm{G}- \\
\mathrm{AN}<\mathrm{A}-\mathrm{AN}\end{array}$} \\
\hline & SD & $\pm 0,48$ & $\pm 0,66$ & $\pm 0,51$ & & & & \\
\hline & $95 \%-K I$ & $1,70-2,02$ & $2,06-2,37$ & $2,69-3,05$ & & & & \\
\hline Emotionale & M & 1,96 & 2,39 & 2,90 & 5,44 & $0,005^{*}$ & 0,075 & $\mathrm{~K}-\mathrm{AN}<\mathrm{G}-$ \\
\hline Kommunikation nach & SD & $\pm 0,56$ & $\pm 0,62$ & $\pm 0,56$ & & & & $\mathrm{AN}<\mathrm{A}-\mathrm{AN}$ \\
\hline & $95 \%-K I$ & $1,77-2,14$ & $2,24-2,54$ & $2,70-3,09$ & & & & \\
\hline Bindungsfähigkeit an & M & 1,87 & 2,50 & 3,13 & 10,84 & $<0,001^{* * *}$ & 0,139 & K-AN $<$ G- \\
\hline innere Objekte ${ }^{a, b}$ & SD & $\pm 0,54$ & $\pm 0,66$ & $\pm 0,53$ & & & & AN $<$ A-AN \\
\hline & $95 \%-K I$ & $1,69-2,05$ & $2,34-2,66$ & $2,94-3,31$ & & & & \\
\hline Bindungsfähigkeit an & M & 2,11 & 2,69 & 3,26 & 3,27 & $0,041^{*}$ & 0,046 & $\mathrm{~K}-\mathrm{AN}<\mathrm{G}-$ \\
\hline äußere Objekte ${ }^{a, b, c}$ & SD & $\pm 0,74$ & $\pm 0,82$ & $\pm 0,52$ & & & & $A N<A-A N$ \\
\hline & $95 \%-K I$ & $1,86-2,36$ & $2,49-2,88$ & $3,08-3,44$ & & & & \\
\hline Gesamtwert ${ }^{\mathrm{a}, \mathrm{b}}$ & M & 1,91 & 2,41 & 2,89 & 7,09 & $<0,001^{* * *}$ & 0,096 & $\mathrm{~K}-\mathrm{AN}<\mathrm{G}-$ \\
\hline & SD & $\pm 0,45$ & $\pm 0,57$ & $\pm 0,44$ & & & & AN $<$ A-AIN \\
\hline & $95 \%-K I$ & $1,77-2,06$ & $2,27-2,55$ & $2,73-3,04$ & & & & \\
\hline $\begin{array}{l}K \text { K-AN keine selbstbericht } \\
\text { prägte selbstberichtete } \\
\text { telwert, SD Standardabw } \\
\text { Signifikante Einflüsse vor } \\
{ }^{*} p<0,05 \text {; }{ }^{* *} p<0,01 \text {; }\end{array}$ & $\begin{array}{l}\text { ete (anorek } \\
\text { nnorektisch } \\
\text { eichung, } 9 \\
\text { Kovariater } \\
{ }^{*} p<0,001\end{array}$ & $\begin{array}{l}\text { che) Essstör } \\
\text { Essstörungss } \\
\text { b-KI } 95 \% \text {-Ko } \\
\text { f Subskalen }\end{array}$ & $\begin{array}{l}\text { ssymptoma } \\
\text { iptomatik, } A \\
\text { denzinterval } \\
\text { d Gesamtw }\end{array}$ & $\begin{array}{l}\text { G-AN gerin } \\
\text { OVA , analy } \\
\text { If Freiheitsgr } \\
{ }^{a} \text { Alter, }{ }^{b} \text { Vord }\end{array}$ & $\begin{array}{l}\text { e, } F \text { Prü } \\
\text { nnosen, }\end{array}$ & $\begin{array}{l}\text { lorektische) Es } \\
\text { JCOVA „multi } \\
t \text { Effektstärke, } \\
\text { apeutische (V }\end{array}$ & $\begin{array}{l}\text { gssymptomatik, } A- \\
\text { analysis of covarianc } \\
\text { es Eta-Quadrat, } p= \\
\text { indlungen }\end{array}$ & $\begin{array}{l}\text { N ausge- } \\
e^{\prime \prime}, M \text { Mit- } \\
\text { ignifikanz }\end{array}$ \\
\hline
\end{tabular}

einem signifikant positiven Zusammenhang mit den Skalen des EDI-2, bei als gering bis hoch zu klassifizierenden Korrelationen (Zusatzmaterial online: Tab. 5).

\section{Diskussion}

\section{Ergebnisinterpretation und Literaturvergleich}

In der vorliegenden Studie wurde die psychische Struktur gemäß OPD bei weiblichen Adoleszenten und jungen Erwachsenen mit unterschiedlich ausgeprägter anorektischer Essstörungssymptomatik in einer Onlinestichprobe untersucht. Hierbei ergaben sich signifikante Unterschiede bei überwiegend mittleren bis hohen Effektstärken zwischen den 3 Gruppen auf 7 von 8 OPD-SF-Skalen sowie in Bezug auf den OPD-SF-Gesamtwert. Einzig auf der Skala der Regulierung des äußeren Objektbezugs offenbarten sich keine signifikanten Gruppenunterschiede. Es ist denkbar, dass bereits innerhalb der anorektischen Essstörungssymptomatik der Objektbezug so reguliert wird, dass betroffene Probandinnen diesbezüglich keine Probleme wahrnehmen (Reich und v. Boetticher 2017). Zudem zeigten sich geringe bis hohe Zusam- menhänge zwischen unterschiedlichen Dimensionen der OPD-Struktur und essstörungsspezifischer Psychopathologie. Die Durchschnittswerte der OPDSF-Struktur waren in Bezug auf sämtliche Strukturdimensionen und auch den Gesamtwert in allen 3 Gruppen höher als in vergleichbaren Stichproben (Bock et al. 2018; Ehrenthal et al. 2012).

Die Ergebnisse dieser Studie bestätigen nur in Teilen die bisherigen Forschungsbefunde, da die Werte aller Gruppen deutlich über den Vergleichswerten lagen. So gaben beispielsweise Probandinnen der A-AN-Gruppe auf allen OPD-Skalen höhere Werte an als 
Patienten in der Studie von Rohde et al. (2019). Auf einigen OPD-SF-Skalen fanden sich sogar höhere Werte bei Probandinnen der G-AN-Gruppe als bei den Patienten mit manifester Essstörung in der Untersuchung von Rohde et al. (2019). Im Vergleich zu den Kontrollgruppen der Studien von Ehrenthal et al. (2012) sowie Bock et al. (2018) zeigten sich ebenfalls in allen hier erhobenen Gruppen deutlich höhere Werte. $\mathrm{Da}$ diese Unterschiede systematisch sind, stehen die hohen Werte möglicherweise im Zusammenhang mit dem methodischen Vorgehen. Die Daten der vorliegenden Studie wurden im Gegensatz $\mathrm{zu}$ allen bisherigen Studien zum OPD-SF nach Rekrutierung über Plattformen für soziale Medien generiert. Neben den Einschränkungen dieser Erhebungsvariante (s. Abschn. „Stärken und Schwächen“), existieren Spezifika, die die hohen Werte aller Gruppen erklären könnten. Zum einen kommt es mithilfe computergestützter Befragungen zu einer geringeren sozialen Präsenz, die die perzipierte Anonymität vergrößert und zu einer verminderten Bewertungsangst und stärkeren Selbstoffenbarung führt (Taddicken 2009). Berger (2018) argumentiert, dass es anhand des Onlineenthemmungseffekts (Vermeidung physischer Nähe, Blickkontakt und Mimik) zu einer intimeren Kommunikation komme. Zum anderen wird berichtet, dass sozial erwünschtes Antwortverhalten geringer ausgeprägt ist (Taddicken 2009). Bei den Probandinnen handelt es sich um eine Altersgruppe, für die die Nutzung digitaler Medien und sozialer Netzwerke einen hohen Stellenwert einnimmt (Lepkowsky und Arndt 2019) und für die die perzeptive Anonymität und der damit verbundene Onlineenthemmungseffekt eine größere Rolle als für erwachsene Probanden spielen könnten. Zum anderen könnten - aufgrund des ausgeprägten Perfektionismus sowie der vermeintlichen sozialen Anpassung und des damit verbundenen sozial erwünschten Antwortverhaltens (Dahlenburg et al. 2019) - bisherige Erhebungen im klassischen „face-to-face setting" Verzerrungen aufweisen und die OPD-SF-Werte unterschätzen. Darüber hinaus könnte die Erhebung über soziale
Medien, einschließlich spezieller Foren und Gruppen, die sich mit Essstörungen beschäftigen, $\mathrm{zu}$ einem Selektionsbias geführt haben, der besonders auf die Gruppe ohne selbstberichtete Essstörungssymptomatik zutrifft (K-AN). Letztlich sind diese Thesen jedoch aus den vorliegenden Daten nicht belegbar, weshalb weitere Untersuchungen notwendig sind, bevor endgültige Aussagen in Bezug auf Effekte von Onlineerhebungen über soziale Netzwerke getroffen werden können.

\section{Stärken und Schwächen}

Zunächst ist $\mathrm{zu}$ erwähnen, dass es sich bei der vorliegenden Studie um eine Querschnittsstudie handelt, wodurch nur begrenzt Rückschlüsse auf bestimmte Stadien von Essstörungen oder psychische Struktur - Aspekte, die sich longitudinal verändern - gezogen werden können. Um langfristige und kausale Aussagen über die Entwicklung der psychischen Struktur und erhöhter Essstörungssymptomatik sowie deren Zusammenwirken treffen zu können, benötigt es längsschnittliche Studien. Kritisch betrachtet werden muss ferner die geringe Fallzahl bei den Probandinnen der A-AN- sowie K-AN-Gruppe im Vergleich zu der G-AN-Gruppe. Zudem muss bedacht werden, dass die Kerndiagnosekriterien einer Anorexia nervosa mithilfe von Fragen operationalisiert wurden, die an das SIAB angelehnt sind, für dessen abgewandelte Form jedoch keine Validierung stattgefunden hat. Insofern können - aufgrund des methodischen Designs - keine Aussagen zur psychischen Struktur bei klinisch diagnostizierten Patienten mit Anorexia nervosa getätigt werden. Darüber hinaus kann eine mögliche Stichprobenverzerrung aufgrund des „Coverage“-Effekts (d.h., dass bestimmte Gruppen im Internet besser erreichbar sind als andere) nicht ausgeschlossen werden. Dennoch bleibt zu erwähnen, dass insbesondere die Vorteile einer Onlinediagnostik bei bestimmten psychischen Störungsbildern, wie z. B. Essstörungen, beachtet werden müssen. Es handelt sich um ein Störungsbild, das sich typischerweise in der Adoleszenz (Briegel et al. 2018) und damit einer Altersgruppe manifestiert, für die die Nutzung digitaler Medien selbstverständlich erscheint (Lepkowsky und Arndt 2019). Zum anderen weisen Patienten mit Essstörungen häufig eine Krankheitsverleugnung auf, zeigen sich angepasst und antworten stark sozial erwünscht, weshalb hier die perzeptive Anonymität und der damit verbundene Onlineenthemmungseffekt einen positiven Effekt haben könnten.

\section{Fazit für die Praxis}

\section{- Neben dem manifesten Ausbruch einer Essstörungssymptomatik kann es zu subklinischen Formen essge- störten Verhaltens kommen. \\ - Es zeigen sich deutliche Einschrän- kungen in der psychischen Struktur bei Probandinnen ohne klinisch diagnostizierte, jedoch mit selbstbe- richteter Essstörungssymptomatik. \\ - Im Rahmen von Onlinemethodiken könnten Betroffene niedrigschwellig erreicht sowie Interventionen und Hilfsangebote offeriert werden, die neben der leitliniengerechten Therapie auch auf die strukturellen Defizite fokussieren.}

\section{Korrespondenzadresse}

\section{Dr. Lea Sarrar}

Fakultät Naturwissenschaften, Department Psychologie, MSB Medical School Berlin Rüdesheimer Str. 50, 14197 Berlin, Deutschland lea.sarrar@medicalschool-berlin.de

Funding. Open Access funding provided by Projekt DEAL.

\section{Einhaltung ethischer Richtlinien}

Interessenkonflikt. A. Obbarius und L. Sarrar geben an, dass kein Interessenkonflikt besteht.

Alle beschriebenen Untersuchungen wurden mit Zustimmung der zuständigen Ethik-Kommission der MSB Medical School Berlin (Kennzeichen: MSB-2019/5), im Einklang mit nationalem Recht sowie gemäß der Deklaration von Helsinki von 1975 (in der aktuellen, überarbeiteten Fassung) durchgeführt. Zudem erfolgte das beschriebene Vorgehen in Konformität mit der Datenschutz-Grundverordnung. Sämtliche Probandinnen bzw. Sorgeberechtigten bestätigten ihr Einverständnis zur Studienteilnahme. 
Open Access. Dieser Artikel wird unter der Creative Commons Namensnennung 4.0 International Lizenz veröffentlicht, welche die Nutzung, Vervielfältigung, Bearbeitung, Verbreitung und Wiedergabe in jeglichem Medium und Format erlaubt, sofern Sie den/die ursprünglichen Autor(en) und die Quelle ordnungsgemäß nennen, einen Link zur Creative Commons Lizenz beifügen und angeben, ob Änderungen vorgenommen wurden.

Die in diesem Artikel enthaltenen Bilder und sonstiges Drittmaterial unterliegen ebenfalls der genannten Creative Commons Lizenz, sofern sich aus der Abbildungslegende nichts anderes ergibt. Sofern das betreffende Material nicht unter der genannten Creative Commons Lizenz steht und die betreffende Handlung nicht nach gesetzlichen Vorschriften erlaubt ist, ist für die oben aufgeführten Weiterverwendungen des Materials die Einwilligung des jeweiligen Rechteinhabers einzuholen.

Weitere Details zur Lizenz entnehmen Sie bitte der Lizenzinformation auf http://creativecommons.org/ licenses/by/4.0/deed.de.

\section{Literatur}

Arbeitskreis OPD (2014) OPD-2 Operationalisierte Psychodynamische Diagnostik: Das Manual für Diagnostik und Therapieplanung. Huber, Bern

Arbeitskreis OPD-KJ-2 (2014) Operationalisierte Psychodynamische Diagnostik im Kindes- und Jugendalter. Grundlagen und Manual. Huber, Bern

Berger T (2018) Die therapeutische Beziehung in internetbasierten Behandlungsansätzen. In: Bents $\mathrm{H}$, Kämmerer A (Hrsg) Psychotherapie und Würde: Herausforderung in der psychotherapeutischen Praxis. Springer, Berlin, Heidelberg, S 105-118

Bock A, Sevecke K, Huber $E$, Weil $P$, Ehrenthal JC (2018) Der OPD-Strukturfragebogen (OPDSF) in Anwendung auf eine jugendliche Schul- und klinische Stichprobe: Ergebnisse zu Reliabilität und Validität. Prax Kinderpsychol Kinderpsychiatr 67:674-690

BriegelW, LennerzB, Löffler J,MoßA, Wabitsch M(2018) Essstörungen und Adipositas im Jugendalter. In: Stier B, Weissenrieder N, Schwab KO (Hrsg) Jugendmed. Springer, Berlin, Heidelberg, S279-289

Cohen J (1988) Statistical power analysis for the behavioral sciences. Lawrence Erlbaum, Hillsdale

Dahlenburg SC, Gleaves DH, Hutchinson AD (2019) Anorexia nervosa and perfectionism: a metaanalysis. Int J Eat Disord 52:219-229

Dilling H, Mombour W, Schmidt MH (2011) Internationale Klassifikation psychischer Störungen. Huber, Bern

Ehrenthal J, Dinger U, Horsch L, Komo-Lang $M$, Klinkerfuß M, Grande T, Schauenburg H (2012) Der OPD-Strukturfragebogen (OPD-SF): Erste Ergebnisse zu Reliabilität und Validität. Psychother Psychosom Med Psychol 62:25-32

Fichter M (2015) Epidemiologie der Essstörungen. In: HerpertzS, deZwaan M,ZipfelS(Hrsg) Handbuch Essstörungen und Adipositas. Springer, Berlin, Heidelberg, S45-46

Fichter M, Quadflieg N (1999) Strukturiertes Inventar für Anorektische und Bulimische Essstörungen nach DSM-IV und ICD-10. Hogrefe, Göttingen

Field A (2013) Discovering statistics using IBM SPSS statistics. SAGE, London
Hartmann AS, Thomas JJ, Greenberg JL, Matheny NL, Wilhelm S (2014) A comparison of self-esteem and perfectionism in anorexia nervosa and body Dysmorphic disorder. J Nerv Ment Dis 202:883-888

HuberE, BockA, Hans M, WieserE, Steinmayr-Gensluckner M, Sevecke K, Benecke C (2017) Emotionserleben, Emotionsregulation und strukturelle Störung bei Jugendlichen. Psychother Forum 22:48-62

Kappel V, Thiel A, Holzhausen M, Jaite C, Schneider N Pfeiffer E, Lehmkuhl U, Salbach-Andrae H (2012) Eating Disorder Inventory-2 (EDI-2). Normierung an einer Stichprobe normalgewichtiger Schüler im Alter von 10 bis 20 Jahren und an Patientinnen mit Anorexia nervosa. Diagnostica 58:127-144

Lepkowsky CM, Arndt S (2019) The Internet: barrier to health care for older adults? Pract Innov 4:124-132

Pace CS, Guiducci V, Cavanna D (2016) Attachment in eating-disordered outpatients with and without borderline personality disorder. J Health Psychol 22:1808-1818

Paul T, Thiel A (2005) EDI-2. Eating disorder inventory-2. Hogrefe, Göttingen

Reich G, v. Boetticher A (2017) Hungern um zu leben - die Paradoxie der Magersucht. Psychodynamische und familientherapeutische Konzepte. Psychosozial-Verlag, Gießen

Rohde J, Hofmann T, Voigt B, Rose M, Obbarius A (2019) Measurement of personality structure by the OPD structure questionnaire can help to discriminate between subtypes of eatingdisorders. Front Psychol 10:2326

Spitzer C (2004) Zum Zusammenhang zwischen OPD-Merkmalen der Persönlichkeitsstruktur und symptombezogenen sowie interpersonalen Behandlungsergebnissen stationärer Psychotherapie. Z Psychosom Med Psychother 50:70-85

Taddicken M (2009) Methodeneffekte von WebBefragungen: Soziale Erwünschtheit vs. Soziale Entkontextualisierung. In: Weichbold M, Bacher J, Wolf C (Hrsg) Umfrageforschung. VS, Wiesbaden, S85-104
Hier steht eine Anzeige. Springer 